\title{
Measuring athletic performance in post-metamorphic fire salamanders
}

\author{
Erica de Rysky, Bisconti Roberta* (1), Chiocchio Andrea and Canestrelli Daniele
}

\begin{abstract}
Objective: Athletic performances are dynamic movements that are physically challenging and often predict individual success in ecological contexts. They stem from a complex integration of multiple phenotypic traits-e.g., morphological, physiological and behavioural — that dictate animal survival and individual fitness. However, directly quantifying athletic performances can be particularly challenging in cryptic, slow-moving species or not very reactive in attitude. Here we present and describe a rapid, simple, and low-cost method to measure athletic performance in post-metamorphic individuals of the fire salamander Salamandra salamandra. While extremely reactive during the larval stage, adult salamanders are, in fact, cryptic and relatively slow-moving.

Results: Forcing terrestrial juveniles to swim under standard, albeit ecologically plausible, laboratory conditions, and using an automatic point-mass tracking tool, we were able to measure maximal and average performance indicators of post-metamorphic individuals. This method avoids inter-individual variation in motivation, as it forces individuals to perform at their best. Moreover, with this method, measures of athletic performance will be directly comparable between larval and terrestrial stages, allowing to study the contribution of carryover effects to the wide range of processes implicated in the eco-evo-devo of athletic performance in salamanders.
\end{abstract}

Keywords: Athletic performance, Fire salamander, Salamandra salamandra, Swimming

\section{Introduction}

Athletic performances are key abilities in animals' life since they predict individual success in ecological contexts $[1,2]$. They consist of dynamic movements that are physically challenging, allowing animals to interact with other conspecific and heterospecific organisms, and with the physical environment [1-3]. These abilities stem from a complex integration of multiple phenotypic traits - e.g., morphological, physiological and behavioural -, that dictate animal survival and fitness in a wide range of contexts [2-4]. Consequently, the comparative study of maximal individual performances in athletic tasks can offer an excellent window on the diversity and evolution of functional phenotypic traits within and among species,

*Correspondence: bisconti@unitus.it

Dipartimento Di Scienze Ecologiche E Biologiche, Università Della Tuscia. Viale Dell'Università S.N.C, 01100 Viterbo, Italy and on the role of athletic abilities in moulding the evolutionary pathways of intra- and interspecific lineages in response to significant changes of their physical and biotic environment [5-8].

Among athletic abilities, locomotor performance has been extensively studied, due to its direct implication in a wide range of ecological tasks, such as predator escape, prey capture, territory defence, reproductive success, dispersal, and so forth $[1,3,6,9,10]$. Traditionally, the bulk of studies investigating the evolution of locomotor traits have been focused on analysing the origin (e.g., convergent vs independent) and the evolutionary trends in animal locomotion along the tree-of-life [1 and reference therein]. However, more recently, increasing attention is being focused on studying inter-individual differences in locomotor performances within and among populations within species [11-13]. In fact, there is an increasing awareness that 
athletic abilities can contribute to shaping the eco-evolutionary processes implicated in the genesis of biogeographic patterns both above and below the species level [14-16]. Among vertebrates, much research effort has been devoted to the study of athletic abilities in the lizards of the genus Anolis [17-19] and cane toad Rhinella marina $[15,20-23]$. The integrative analysis of jumping performances in these study systems (based on biomechanical, physiological, and morphometric traits; [22, 24]) highlighted an unexpected amount of inter-individual diversity in performance abilities and suggested a major role of these abilities in shaping the evolution both of dispersal capacities and the species range, over multiple spatial and temporal scales $[19,22,23$, $25]$. As a result, increasing insights on the eco-evolutionary role of inter-individual differences in animal performances traits are coming from this kind of studies. However, performing experimental studies can be in many cases hampered by species-specific lifestyles, such as in species that are cryptic and tend not to react impulsively to external stimuli. In these species, identifying and directly quantifying genuinely informative athletic performance traits could be challenging.

The European fire salamander Salamandra salamandra (Fig. 1A) is a urodele amphibian widely distributed across much of the Western Palaearctic, where it inhabits mixed, moist deciduous forests from the sea level up to $2500 \mathrm{~m}$ [26, 27]. It is a nocturnal and crepuscular dweller, mostly active on rainy nights, spending daytime under moisty woods, stones or the leaflitter [26]. After the metamorphosis, the fire salamander becomes strictly terrestrial, and only females approach water to lay larvae. In the adult phase, this species shows a distinctively cryptic lifestyle, making the study of maximal locomotor performance quite difficult. Therefore, when found above ground its movements are slow and, even when stimulated by a (natural or mimicked) predator, its defensive behaviour is rather static, characterized by remaining motionless, usually without attempting to escape predator attacks (it possesses antipredator toxin secretions) [28-31]. Nevertheless, measuring maximal locomotor performances is of crucial importance because they are directly implicated in the survival of individuals for prey capture, reproductive success, and dispersal ability.

Here we present a simple, rapid and low-cost method to accurately quantify athletic performance abilities in post-metamorphic fire salamanders. We aim to provide a test that could force this cryptic, and apparently unreactive species, to perform at its extreme limit, allowing the analysis of athletic performance to become part of the extensive body of literature concerning patterns of phenotypic diversity, distribution, and evolution in this species [32, 33].

\section{Main text \\ Methods \\ Sampling and housing}

We collected 78 fire salamander individuals at larval stage immediately after deposition to standardise the entire experimental procedure. With this aim, we monitored breeding sites in the Picentini Mts. (Campania Region, Italy) between March and June in 2019, when the larvae laying usually occurs [27]. Larvae were collected from distinct breeding sites placed at least $20-50 \mathrm{~m}$ from each other, in order to avoid kinship relationships among individuals. All the breeding sites consisted in small ponds along small streams characterized by lowslope and low water velocity/turbulence located in beech forests at around $1000 \mathrm{~m}$ of altitude. Collected larvae were individually housed under controlled and standard conditions [34] in a humid chamber (room temperature $18-20^{\circ} \mathrm{C}$, humidity $65-70 \%$, natural photoperiod: 12-15 h daylight/dark following natural seasonality) at the facilities of the Department of Ecological and Biological Sciences of the University of Tuscia (Viterbo, Lazio, Italy). Salamander larvae were individually housed within pierced plastic baskets $(10 \times 10 \times 10 \mathrm{~cm})$, each with a terracotta saucer as shelter. All plastic baskets were placed collectively within a PVC tank filled with aerated soft water (dechlorinated and demineralized water 1:1) and water temperature $\left(10-12{ }^{\circ} \mathrm{C}\right)$ monitored using a temperature data logger (Hobo Pendant MX2201). Larvae were fed ad libitum three days a week with live tubifex and chironomid larvae. Once metamorphosed, each salamander was housed individually into terrariums made with transparent and micro-perforated plastic boxes $(11.5 \times 11.5 \times 6 \mathrm{~cm})$, with coconut litter and gravel as substrates, a plastic cap containing dechlorinated water, and a piece of cork bark as shelter. Metamorphosed juveniles were fed ad libitum with crickets (Acheta domestica) and mealworms (Tenebrio molitor).

\section{Athletic performance test}

A convenient approach to analyse athletic performance in animals consists of measuring maximal movement performance in response to a stimulus that is perceived as threatening, usually cues of a predator attack [e.g., 35-37]. We performed a series of pilot tests on a group of non-experimental animals (10 individuals) in an attempt to elicit propelling movements. However, even the more invasive version of these tests (i.e., tail pinching) did not elicit consistent and repeatable responses. Most individuals did not react at all, while a few others jumped, u-turned, or sprinted, and the responses were not repeatable over multiple trials. Based on that result, we moved to test individual response to another common threat in fire salamander environments: falling into the water. 

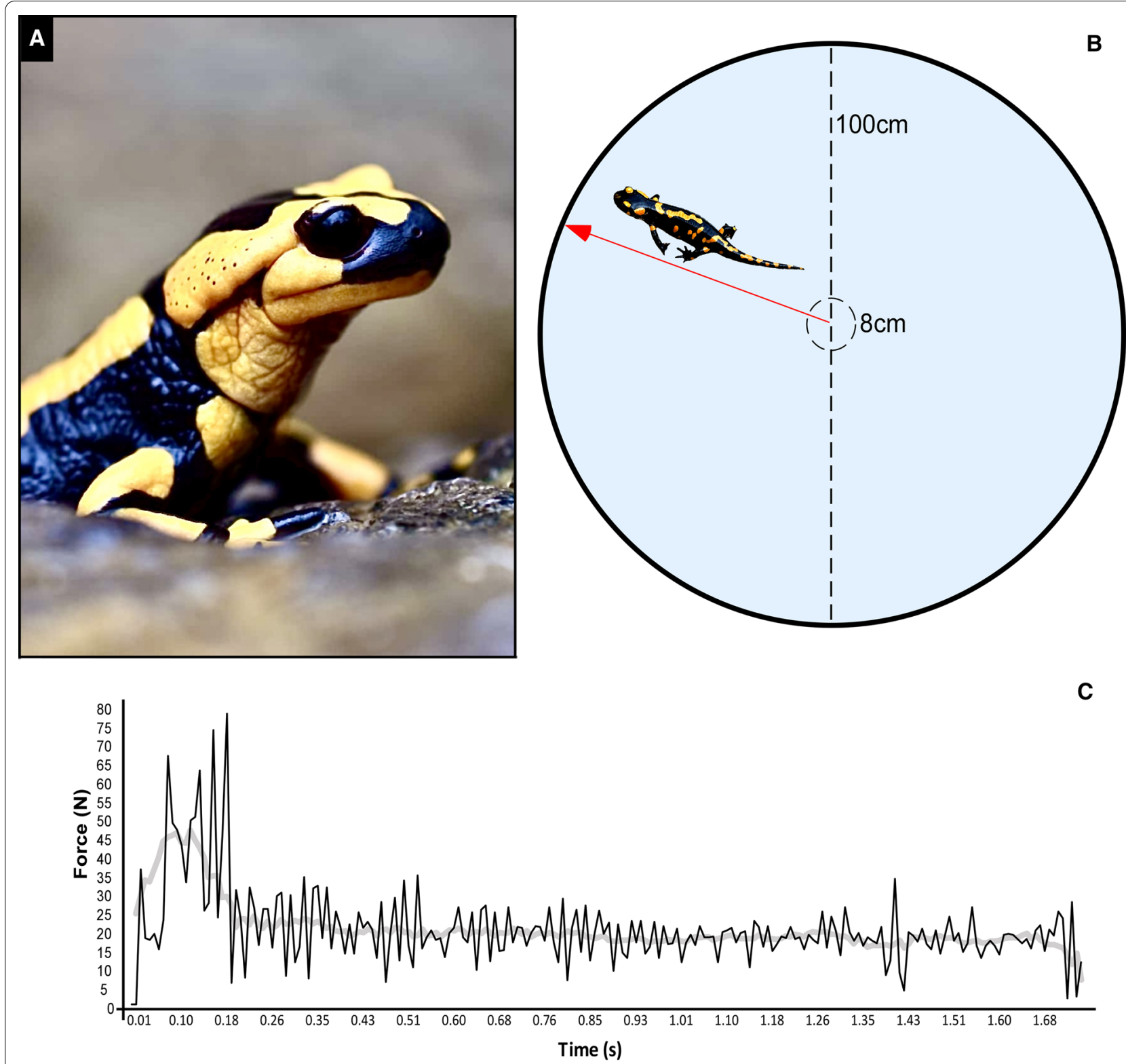

Fig. 1 A The fire salamander Salamandra salamandra (Photo: Grignani G.). B The arena used for the swimming test (salamander is not in scale). C Swimming force profile of an individual as a representative example (black line: raw data; grey line: moving average)

As mentioned above, adult fire salamanders are fully terrestrial. Accidental falls into the water may be fatal, unless the shore is reached promptly. Preliminary trials revealed a consistent response of salamanders to swimming challenges, which is necessary to reflect individual differences.

Swimming performance was measured once for each individual at three months after the metamorphosis to ensure the performance being representative of a fully terrestrial life stage. Swimming tests were carried out under strictly controlled environmental conditions (room temperature $20{ }^{\circ} \mathrm{C}$, humidity $70 \%$; water temperature $15{ }^{\circ} \mathrm{C}$ ) since variability in these conditions might impact individual performance during the test.

The experimental arena consisted of a white circular plastic tank (diameter: $100 \mathrm{~cm}$; water volume: $50 \mathrm{~L}$; water depth: $6.5 \mathrm{~cm}$; see Fig. 1B) to avoid possible "blind spots", where individuals may get stuck or have a foothold to climb out of the water. To standardise the starting phase of the test, when the individuals were released in the tank, the centre of the arena was marked with a small plastic circle placed $1 \mathrm{~cm}$ above the water surface. Thus, 
each individual was gently dropped into the water from the centre of this "launchpad", using a humid spoon. Test duration was set to $2 \mathrm{~min}$. At the end of the test, individuals were weighted with a precision analytical scale $( \pm 0.001 \mathrm{~g})$ to calculate the acceleration force. All individuals were fasted $48 \mathrm{~h}$ before the tests.

Swimming performance tests were video recorded at high frame rates (120 fps), using a GoPro Hero5 camera (at 1080p) placed above the centre of the experimental arena. Videos were then analysed with the software Tracker 5.1.3 (https://physlets.org/tracker). This software allows characterising physical properties of animal movements using automatic point-mass tracking, if high-quality videos are used as input (i.e., enough contrast between tracked objects and the background), together with a measure of the body mass and a known reference scale. We used the tip of the salamander snout as a point mass for tracking and manually set the body mass for each individual and calibrate the scale by setting the diameter of the tank as reference scale. Tracker allows extracting a wide range of performance variables. For illustrative purposes, here, we provide maximum and average values of velocity and acceleration. Potential errors in automatic tracking were checked by eye for each individual by the same operator.

\section{Results and discussion}

All the analysed individuals started swimming immediately after the dive, and continued swimming on the water surface until they reached the edge of the tank (Fig. 1C; Table 1; Fig. 2; an additional movie file shows this in more detail [see Additional file 1]). This behavioural pattern has at least two highly desirable features when testing athletic performances: (1) complete interindividual consistency; (2) a bi-axial configuration of the entire athletic movement. Consistency of the behavioural pattern is essential, as it implies that individual personality does not modulate an individual's

Table 1 Maximum (Max) and mean values of speed and acceleration, and mean time to reach the edge of the experimental arena, measured for the post-metamorphic individuals of Salamandra salamandra analysed

\begin{tabular}{lc}
\hline Variable & Mean \pm S.E \\
\hline Max speed $(\mathrm{cm} / \mathrm{s})$ & $34.21 \pm 1.63$ \\
Mean speed $(\mathrm{cm} / \mathrm{s})$ & $19.19 \pm 0.45$ \\
Relative max speed $(\mathrm{g} / \mathrm{s})$ & $0.07 \pm 0.01$ \\
Relative mean speed $(\mathrm{g} / \mathrm{s})$ & $0.13 \pm 0.01$ \\
Max swimming force $(\mathrm{N})$ & $3949.81 \pm 237.23$ \\
Mean swimming force $(\mathrm{N})$ & $1628.21 \pm 125.63$ \\
Mean time to reach the edge $(\mathrm{s})$ & $3.68 \pm 0.39$ \\
\hline
\end{tabular}

tendency to perform the athletic task at its best, and so it does not act as a confounding experimental factor [2, 38-40]. This argument receives further support by observing that maximum acceleration values are usually reached at the beginning of the test, which is not expected in case personality (e.g., shy vs bold response to a stressful situation) play a significant role affecting motivation. Furthermore, the bi-axial configuration of the movement over the water surface allows ignoring the third (i.e., vertical) axis when deriving acceleration and other physical descriptors of the athletic performance, simplifying the experimental setup substantially.

Once individuals reached the edge of the tank, the behavioural pattern changed significantly, towards a heterogeneous alternation of resting at the edge, swimming and floating phases. Moreover, over multiple testing, consistency of the pattern was observed neither among nor within individuals, which qualifies this second segment of the test as useless for our purposes. It is worth noting that this second segment implies that endurance measures cannot reliably be derived from this test, and the test duration could be reduced from 2 to $1 \mathrm{~min}$ for future use. At the same time, however, this diverse mix of multiple phases might reveal distinct behavioural types along the second segment of the test. If and to what extent behavioural patterns along this segment (e.g., frequency and/or time spent in each phase) might associate with individual personality traits could be an intriguing subject for future experimental scrutiny, given the inherent difficulties in studying personality traits variation in highly cryptic and rather static species.

Measuring animals' athletic performances in cryptic, slow-moving, or difficult-to-track animals could be very challenging [1]. Here, we have described a rapid, simple, and low-cost method to measure athletic performance in fire salamanders under standardized, albeit environmentally realistic, laboratory conditions. By forcing individuals to perform in the aquatic environment, this method successfully avoids the lack of motivation, in line with results obtained with similar approaches in other organisms (e.g., in rodents, [41, 42]). Finally, a point of significant value of the approach presented here stems from the possibility of gaining comparable athletic performance measures between larval and terrestrial stages. Indeed, when in the aquatic medium, post-metamorphic salamanders show a swimming pattern matching the one used by larvae. In light of this, our test will allow us to investigate the contribution of carryover effects across life stages to the wide range of processes implicated in the eco-evo-devo of athletic performance in salamanders [43]. 

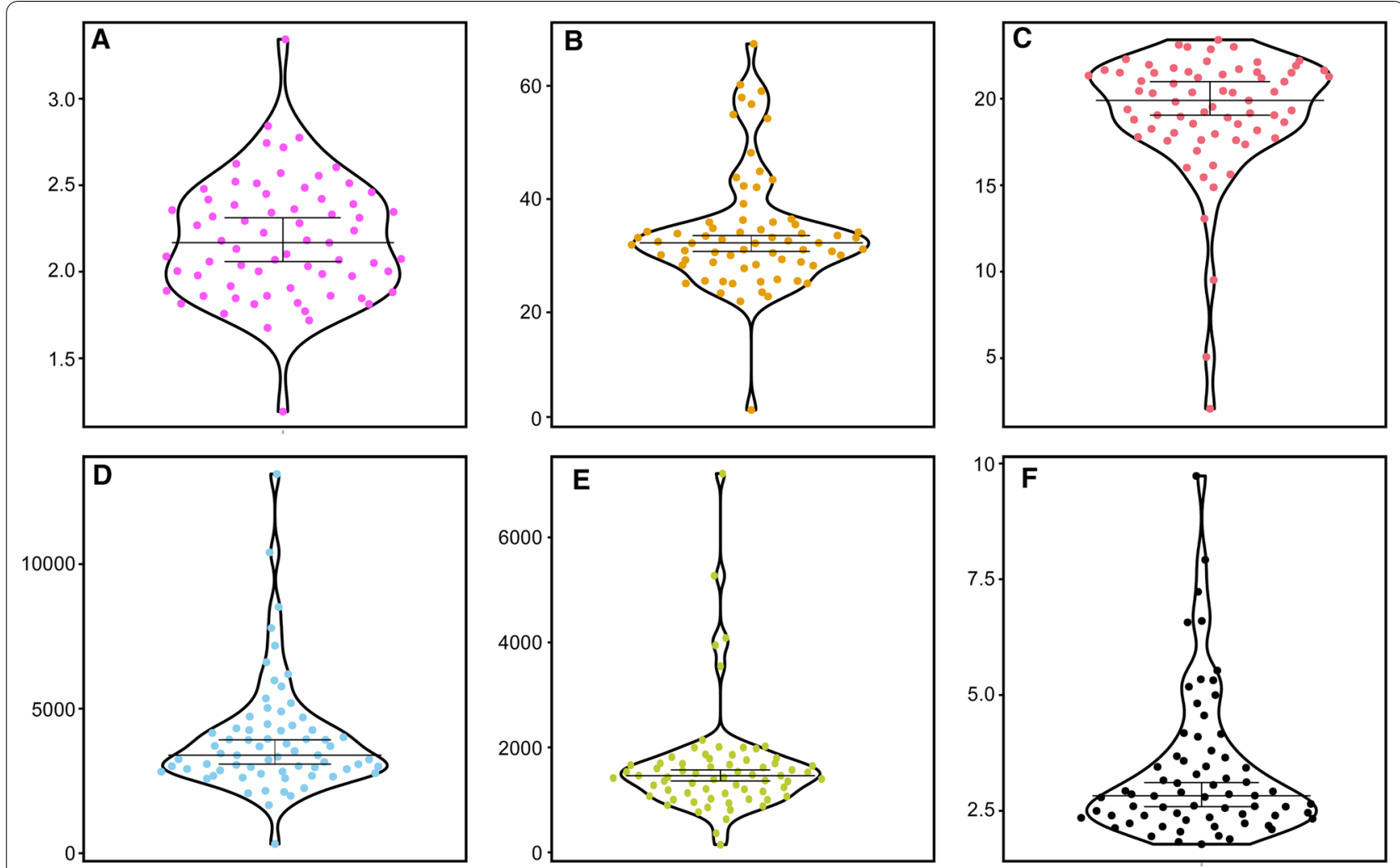

Fig. 2 Violin plots of the measured variables. Horizontal lines display the mean value, and vertical bars show the 95\% confidence interval. A Weight (g); B Max speed (cm/s); C Mean speed (cm/s); D Max swimming force (N); E Mean swimming force (N); $\mathbf{F}$ Time to reach the edge (s). The graphs were drawn using the web app PlotsOfData [44] and the software Canvas 11 (ACD Systems of America, Inc.)

\section{Limitations}

- The method does not allow to measure the endurance of locomotor effort.

- The automatic tracking of the focal subject is only applicable when high-quality videos are used as input (i.e., enough contrast between tracked objects and the background).

\section{Supplementary Information}

The online version contains supplementary material available at https://doi. org/10.1186/s13104-021-05808-0.

Additional file1: Video-tracking of the swimming performance test of a post-metamorphic fire salamander Salamandra salamandra, obtained by using Tracker 5.1.3.

\section{Acknowledgements}

We are grateful to Giacomo Grignani and Giada Spadavecchia for their help with sample collection and experiments.

\section{Authors' contributions}

DC conceived and designed the study. EdR and AC carried out field sampling and mitotype assignment. EdR and RB performed the experiments. DC and
EdR analysed the data. DC, AC, EdR and RB drafted the manuscript. All authors read and approved the manuscript.

\section{Funding}

This research was funded by the University of Tuscia.

\section{Availability of data and materials}

The datasets used during the current study available from the corresponding author on reasonable request.

\section{Declarations}

Ethics approval and consent to participate

All experiments complied with the Guidelines of the European Union Council and were approved by the Ethical Committee of the Tuscia University for the use of live animals (D.R. n. 677/16 and D.R. 644/17). Permission to temporarily house amphibians was granted by Local Health and Veterinary Centre, with license code 050VT427. All procedures were performed under the approval of the Institute for Environmental Protection and Research 'ISPRA' (protocol \# 23501), Ministry of Environment'MATTM' (protocol \#8275) and Regione Campania (protocol \#0203190).

\section{Consent to publication}

Not applicable.

\section{Competing interests}

Not applicable. 
Received: 24 May 2021 Accepted: 11 October 2021

Published online: 26 October 2021

\section{References}

1. Biewener AA, Patek SN. Animal locomotion. 2nd ed. Oxford: Oxford University Press; 2018.

2. Careau V, Garland T. (2012) Performance, personality, and energetics: correlation, causation, and mechanism. Physiol Biochem Zool. 2012;85(6):543-71.

3. Irschick DJ, Higham TE. Animal athletes: an ecological and evolutionary approach. Oxford: Oxford University Press; 2016.

4. Lailvaux SP, Husak JF. Life-history of whole-organism performance. Q Rev Biol. 2014;89(4):285-318.

5. Finkler MS, Claussen DL. Influence of temperature, body size, and inter-individual variation on forced and voluntary swimming and crawling speeds in Nerodia sipedon and Regina septemvittata. J Herpetol. 1999;33(1):62-71.

6. Husak JF, Fox SF. Field use of maximal sprint speed by collared lizards (Crotaphytus collaris): compensation and sexual selection. Evolution. 2006;60(9):1888-95.

7. Irschick DJ, Meyers JJ, Husak JF, Le Galliard J-F. How does selection operate on whole organism functional performance capacities? A review and synthesis. Evol Ecol Res. 2008;10(2):177-96.

8. O'Donnell MK, Deban SM. The effects of roughness and wetness on Salamander Cling performance. Integr Comp Biol. 2020;60(4):840-51.

9. Irschick DJ, Losos JB. A comparative analysis of the ecological significance of locomotor performance in Caribbean Anolis lizards. Evolution. 1998;52:219-26.

10. Fitzpatrick BM, Benard MF, Fordyce JA. Morphology and escape performance of tiger salamander larvae A. tigrinum mavortium. J Exp Zool. 2003:297A:147-59.

11. Elnitsky MA, Claussen DL. The effects of temperature and inter-individual variation on the locomotor performance of juvenile turtles. J Comp Physiol B. 2006;176(6):497-504.

12. Rob SJ, Walter I, Seebacher F. Variation in expression of calcium-handling proteins is associated with inter-individual differences in mechanical performance of rat (Rattus norvegicus) skeletal muscle. J Exp Biol. 2011;214(21):3542-8.

13. Careau V, Biro PA, Bonneaud C, Fokam EB, Herrel A. Individual variation in thermal performance curves: swimming burst speed and jumping endurance in wild-caught tropical clawed frogs. Oecologia. 2014;175:471-80.

14. Irschick DJ, Vitt LJ, Zani PA, Losos JB. A comparison of evolutionary radiations in Mainland and West Indian Anolis lizards. Ecology. 1997:78(7):2191-203.

15. Kosmala G, Christian K, Brown G, Shine R. Locomotor performance of cane toads differs between native-range and invasive populations. R Soc Open Sci. 2017;4: 170517.

16. Llewelyn J, Phillips BL, Alford RA, Schwarzkopf L, Shine R. Locomotor performance in an invasive species: cane toads from the invasion front have greater endurance, but not speed, compared to conspecifics from a long-colonised area. Oecologia. 2010;162(2):343-8.

17. Irschick DJ. Effects of behavior and ontogeny on the locomotor performance of a West Indian lizard Anolis lineatopus. Funct Ecol. 2000;14(4):438-44.

18. Toro E, Herrel A, Irschick DJ. The evolution of jumping performance in Caribbean Anolis lizards: solutions to biomechanical trade-offs. Am Nat. 2004;163:844-56.

19. Vanhooydonck B, Herrel A, Van Damme R, Irschick DJ. The quick and the fast: the evolution of acceleration capacity in Anolis lizards. Evolution. 2006:60(10):2137-47.

20. Phillips BL, Brown GP, Webb JK, Shine R. Invasion and the evolution of speed in toads. Nature. 2006;439:803.

21. Cabrera-Guzmán E, Crossland MR, Brown GP, Shine R. Larger body size at metamorphosis enhances survival, growth and performance of young cane toads (Rhinella marina). PLoS One. 2013;8: e70121.
22. Hudson CM, McCurry MR, Lundgren P, McHenry CR, Shine R. Constructing an invasion machine: the rapid evolution of a dispersal-enhancing phenotype during the cane toad invasion of Australia. PLoS One. 2016;11: e0156960.

23. Hudson CM, Vidal-García M, Murray TG, Shine R. The accelerating anuran: evolution of locomotor performance in cane toads (Rhinella marina, Bufonidae) at an invasion front. Proc R Soc Biol Sci. 1938;2020(287):20201964.

24. Irschick DJ, Vanhooydonck B, Herrel A, Meyers J. Intraspecific correlations among morphology, performance and habitat use within a green anole lizard (Anolis carolinensis) population. Biol J Linn Soc. 2005;85(2):211-21.

25. Phillips BL, Brown GP, Shine R. Evolutionarily accelerated invasions: The rate of dispersal evolves upwards during the range advance of cane toads. J Evol Biol. 2010;23(12):2595-601.

26. Lanza B, Nistri A. Anfibi d'Italia. Ministero dell'Ambiente e della Tutela del Territorio e del Mare, ISPRA. Modena: Grandi and Grandi Editori; 2009.

27. Sparreboom M. Salamanders of the old world: the salamanders of Europe, Asia and northern Africa. Netherlands: KNNV Publishing, Brill; 2014.

28. Brodie ED Jr, Johnson JA, Dood CK Jr. Immobility and defensive behaviour in salamanders. Herpetologica. 1974;30(1):79-85.

29. Brodie ED. Salamanders antipredator postures. Copeia. 1977;1977(3):523-35.

30. Lüddecke T, Schulz S, Steinfartz S, Vences M. A salamander's toxic arsenal: review of skin poison diversity and function in true salamanders, genus Salamandra. Sci Nat. 2018:105(9-10):56.

31. Preißler K, Gippner S, Lüddecke T, Krause ET, Schulz S, Vences M, Steinfartz S. More yellow more toxic? Sex rather than alkaloid content is correlated with yellow coloration in the fire salamander. J Zool. 2019;308(4):293-300.

32. Steinfartz $S$, Veith $M$, Tautz D. Mitochondrial sequence analysis of Salamandra taxa suggests old splits of major lineages and postglacial recolonizations of Central Europe from distinct source populations of Salamandra salamandra. Mol Ecol. 2000;9(4):397-410.

33. Bisconti R, Porretta D, Arduino P, Nascetti G, Canestrelli D. Hybridization and extensive mitochondrial introgression among fire salamanders in peninsular Italy. Sci Rep. 2018;8:13187.

34. Staniszewski M. Amphibians in captivity. Neptune city: T.F.H. Publications; 1995.

35. Diamond KM, Lagarde R, Schoenfuss HL, Walker JA, Ponton D, Blob RW. Relationship of escape performance with predator regime and ontogeny in fishes. Biol J Linn Soc. 2019;127(2):324-36.

36. Freymiller GA, Whitford MD, Higham TE, Clark RW. Escape dynamics of free-ranging desert kangaroo rats (Rodentia: Heteromyidae) evading rattlesnake strikes. Biol J Linn Soc. 2019;127(1):164-72.

37. Baxter-Gilbert J, Riley JL, Whiting MJ. Runners and fighters: clutch effects and body size drive innate antipredator behaviour in hatchling lizards. Behav Ecol Sociobiol. 2018;72(6):1-9.

38. Losos JB, Cree DA, Schulte JA. Cautionary comments on the measurement of maximum locomotor capabilities. J Zool. 2002;258(1):57-61.

39. Irschick DJ. Measuring performance in nature: implications for fitness variation within populations. Integr Comp Biol. 2003;43(3):396-407.

40. Astley HC, Abbott EM, Azizi E, Marsh RL, Roberts TJ. Chasing maximal performance a cautionary tale from the celebrated jumping frogs. J Exp Biol. 2013:216(Pt 21):3947-53.

41. Can A, Dao DT, Arad M, Terrillon C, Piantadosi SC, Gould TD. The Mouse Forced Swim Test. J Vis Exp. 2012;59: e3638.

42. Suvrathan A, Tomar A, Chattarji S. Effects of chronic and acute stress on rat behaviour in the forced-swim test. Stress. 2010;13(6):533-40.

43. Moore MP, Martin RA. On the evolution of carry-over effects. J Anim Ecol. 2019;88(12):1832-44.

44. Postma M, Goedhart J. PlotsOfData - a web app for visualizing data together with their summaries. PLOS Biol. 2019;17(3): e3000202.

\section{Publisher's Note}

Springer Nature remains neutral with regard to jurisdictional claims in published maps and institutional affiliations. 